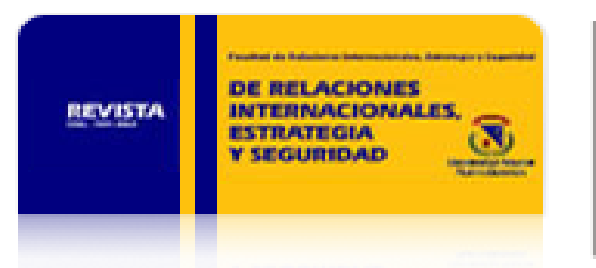

Revista de Relaciones Internacionales, Estrategia y Seguridad

ISSN: 1909-3063

cinuv.relinternal@unimilitar.edu.co

Universidad Militar Nueva Granada

Colombia

Montero Moncada, Luis Alexander

Política exterior de Seguridad Estadounidense en la post-Guerra Fría y el Mundo Post 11 de

Septiembre

Revista de Relaciones Internacionales, Estrategia y Seguridad, vol. 1, núm. 2, julio-diciembre, 2006,

pp. 105-120

Universidad Militar Nueva Granada

Bogotá, Colombia

Disponible en: http://www.redalyc.org/articulo.oa?id=92710206

- Cómo citar el artículo

Número completo

- Más información del artículo

- Página de la revista en redalyc.org

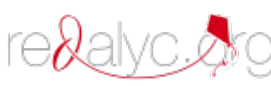

Sistema de Información Científica

Red de Revistas Científicas de América Latina, el Caribe, España y Portugal

Proyecto académico sin fines de lucro, desarrollado bajo la iniciativa de acceso abierto 


\section{POLÍTICA EXTERIOR DE SEGURIDAD ESTADOUNIDENSE EN LA POST GUERRA FRÍA Y EL MUNDO POST 11 DE SEPTIEMBRE}

Por: Luis Alexander Montero Moncada ${ }^{1}$

\section{RESUMEN}

La política exterior de seguridad estadounidense ha sido determinante para la configuración de distintos órdenes internacionales. Durante la Guerra Fría, Estados Unidos construyó una agenda de seguridad basada en la confrontación con el enemigo socialista a partir de la amenaza nuclear. Al finalizar la Guerra Fría, Estados Unidos diseñó una nueva agenda basada en una remodelación del medio ambiente

1 Profesor Departamento Humanidades Universidad Central. Politólogo Universidad Nacional. Magíster en Análisis de problemas políticos, económicos e internacionales contemporáneos, Instituto de Estudios Políticos de París - Universidad Externado - Ministerio de Relaciones Exteriores. Correspondencia: Calle 4538 A-16 Bloque 2 Apto. 204. Bogotá. Correo electrónico: alexander.montero@gmail.com 
internacional, una adecuada respuesta al espectro completo de las crisis y la preparación para un futuro incierto. Aunque esta agenda pretendió responder a los retos de un mundo nuevo, fracasó ante los ataques del 11 de septiembre de 2001. Después de los ataques terroristas, la agenda de seguridad contemporánea crea una nueva definición de la seguridad y redefine las amenazas de Estados Unidos, pero no crea un paradigma nuevo, lo cual genera serias dudas sobre la adecuada aplicación de una política de seguridad.

Palabras clave: Seguridad, Política Exterior, Terrorismo, Amenazas, Agenda, Estrategia Militar.

\section{ABSTRACT}

The Foreign Security Policy of United States has been determinant in the configuration of different international orders. During Cold War, United States built a based- anti - socialist action security agenda supported in the nuclear challenge. At the end of Cold War, United States designed a new agenda supported on shaping the international environment, responding to the full spectrum of crises and Preparing for an uncertain future. This agenda tried to respond to different challenges in a new world; nevertheless, it failed before 11.09.01 attacks. After attacks, the contemporary security agenda makes a new security definition and redesigns the United States challenges, but it doesn't built a new security paradigm, and for this reason, this agenda offers serious questions about an adequate applications of any security policy.

Key words: Security, Foreign Policy, Terrorism, Challenges, Agenda, Military strategy. 


\section{INTRODUCCIÓN}

Durante cerca de cuarenta años, las relaciones internacionales entre Estados Unidos y América Latina se enmarcaron bajo las premisas militares y políticas de la Guerra Fría que concebían a la región como un espacio para lograr una acción conjunta en contra del socialismo, bajo las particularidades de los ejércitos y los gobiernos latinoamericanos.

Sin embargo, al finalizar la Guerra Fría surgen nuevas prioridades en la agenda exterior de seguridad estadounidense y América Latina, vista bajo una óptica diferente a la que existía hasta entonces. Dentro de esta nueva agenda exterior de seguridad que elaboró Estados Unidos, existían elementos críticos que afectaban intereses estadounidenses vitales y secundarios, referidos a la estabilidad económica, política o social de la región, con problemáticas diversas como narcotráfico, conflictos internos y regionales, proliferación de tecnología militar de destrucción masiva, amenazas transnacionales y desastres humanitarios como migraciones incontroladas que se encontraban presentes y aún hoy existen, en América Latina.

Esta agenda exterior de seguridad, sólo tuvo una década de vida útil y luego del 11 de septiembre de 2001, se vio modificada, al menos en parte, por el discurso antiterrorista mundial, en el cual Estados Unidos identificó una serie de aliados para enfrentar a unos posibles Estados hostiles, hospederos de agrupaciones terroristas.

El presente artículo pretende hacer un paralelo analítico entre estos tres momentos de la seguridad estadounidense, identificando los principales componentes de cada agenda exterior de seguridad y las implicaciones que tuvieron en el terreno de las Relaciones Internacionales. 
En cuanto al eje teórico de discusión, se puede decir que la apreciación de la política exterior de seguridad de Estados Unidos bajo el análisis de las Relaciones Internacionales como ciencia, resulta escasa si se toma una sola corriente teórica, por cuanto no aseguraría universalidad en la tarea. No se puede encontrar una única corriente teórica que dé cuenta real de todo el universo de componentes, interacciones y alcances de la política exterior estadounidense; sólo pueden servir como lentes, desde los cuales se ha de analizar el objeto de estudio, y encontrar elementos coincidentes, difusos o absolutamente lejanos con la teoría o teorías utilizadas.

Es difícil suponer o asegurar que la Política Exterior, incluso en cuanto a la seguridad interna y externa, tiene muchos más elementos comunes a una teoría en particular. Todas las discusiones teóricas encierran elementos comunes y elementos opuestos e incluso, el hecho de que la política exterior estadounidense se haya comportado igual en sus acciones de manera independiente de la filiación partidista del presidente o de la composición del congreso, hace infructuoso cualquier intento de encasillamiento.

Sin embargo, para el caso de este estudio y teniendo en cuenta las limitaciones, resulta conveniente una mayor concentración en el neorrealismo como teoría de las relaciones internacionales, para analizar la política exterior de seguridad de Estados Unidos y el despliegue de sus fuerzas. Esta decisión, se fundamente básicamente en cuatro hechos:

El primero es que el neorrealismo, si bien puede encontrar sus primeras luces a fines de los años 70, fue la teoría de las relaciones internacionales que mayor desarrollo logró, y llegó a ser casi hegemónica en los años que antecedieron al desplome de la Unión Soviética, al analizar un sistema internacional absolutamente bipolar; fue la teoría de mayor adecuación para el nuevo sistema (Buzan, 1993: 327-352). Sin embargo, el hecho de que después del fin de la guerra fría, la política exterior de Estados Unidos se pueda analizar aún bajo ésta óptica, produce inquietud y dudas sobre el verdadero cambio en la esencia de la política exterior estadounidense. Aunque se produjo un cambio en el discurso, no lo hubo en la disposición de las tropas y por consiguiente, el espíritu de la política exterior sigue siendo prácticamente el mismo de la Guerra Fría.

En segundo lugar, los conceptos centrales de la política exterior de seguridad de Estados Unidos, representada formalmente en el Quadrennial 
Defense Review y en la Iniciativa de Defensa Estratégica, en la post guerra fría y luego del 11 de septiembre de 2001, tratan sobre las categorías centrales del dominio del neorrealismo. Tal como se analiza más adelante, los principales conceptos coincidentes son seguridad, anarquía, supervivencia, balance de poder, poder, interacción con la economía y recurso militar y tecnológico.

En tercer lugar, en el neorrealismo y en la política exterior de seguridad estadounidense, la interacción de los elementos conceptuales estructurales propone un estado natural de constante confrontación en el sistema internacional.

El cuarto punto es una teoría que se ha ido adaptando a los cambios, con la misma rapidez que ellos ocurren en el sistema internacional actual. No es una teoría de lento desarrollo, como quedó demostrado en la coyuntura del final de la guerra fría y en los años 90. Si bien el neorrealismo que desarroIló Waltz en su libro Teoría de la Política Internacional, contenía un análisis y unos principios propios y pertinentes para el mundo de la guerra fría que lo condujo como consecuencia lógica, a su profunda crisis y necesidad de revisión, logró sobreponerse a esta crisis e introdujo en la disciplina de las Relaciones Internacionales, nuevas variantes del realismo que retomaron sus principios centrales como seguridad, estabilidad hegemónica, poder militar e interacción con la economía, y los adecuó a las nuevas condiciones y retos teóricos de la política internacional contemporánea, permitiendo tener a disposición, una serie de análisis sobre un mundo cambiante y que conservaran de paso, una línea teórica definida.

Es importante señalar que el concepto de estrategia que se incluye en este texto, es en gran medida la que desarrolló André Beaufre en su libro Introduction à la Stratégie, cuando se refiere a la estrategia general y en este caso, a la estrategia general militar. Según Beaufre, esta última se subordina a la estrategia total que conduce la guerra e involucra estrategias militares, económicas, políticas y diplomáticas, y que debe combinar de la mejor forma, los recursos y las acciones terrestres, aéreas y navales, según el análisis de los lineamientos centrales de la agenda de seguridad y las condiciones generales del terreno (Beaufre, 1985: 24-26), o en este caso, del Área de Operaciones. En otras palabras, la estrategia general militar es el espacio donde se concibe la idea militar, según los parámetros emanados de la estrategia total 


\section{LA SEGURIDAD Y DEFENSA DE ESTADOS UNIDOS EN LOS AÑOS 90. RETOS Y PREOCUPACIONES A ESCALA MUNDIAL}

Durante la guerra fría, la principal preocupación de la política exterior de seguridad de Estados Unidos, era el enfrentamiento nuclear con el bloque soviético, que perdió toda la relevancia que había tenido desde 1947. La Unión Soviética como principal contendiente, y en consecuencia, la mayor amenaza a la seguridad de Estados Unidos, colapsó y se perdió así, el núcleo alrededor del cual se construyó y desarrolló toda la estrategia de defensa estadounidense durante cerca de cuarenta años.

En la posguerra fría, la percepción de la amenaza se amplió y dejó de ser exclusivamente un objetivo identificado, para dar paso a amenazas que provenían de sectores o actores menos delimitados (Fontana, 1997:11). Además, se introdujo el ámbito doméstico que elevó en forma considerable, la participación de los aspectos económicos y políticos dentro de la agenda (Sweedler, 1999:408), haciendo que el componente militar modificara el protagonismo que tuvo durante la guerra fría. Se puede decir entonces, que pese a que los aspectos meramente militares, podían continuar siendo importantes para enfrentar las amenazas armadas, la importancia o mejor aún, la supremacía de lo militar se vio matizada por las dimensiones económicas y políticas de la nueva agenda de seguridad.

\subsection{Cambio en el concepto de seguridad en los años 90}

El incremento de la importancia de lo económico y lo político dentro de la agenda exterior de seguridad de Estados Unidos, se puede ver desde la discusión planteada por Daniel Kaufman, Jeffrey Mckitrick y Thomas Leney en el artículo How to Analize National Security, publicado en 1985 y que trata sobre la conceptualización de la seguridad nacional estadounidense durante y después de la guerra fría.

Estos autores afirman que por una parte, existe la perspectiva estrecha o visión tradicionalista (Bárcenas, 2000:15), de la seguridad nacional, referida a la defensa militar ante amenazas militares y que privilegia la dimensión internacional de la seguridad. Este fue el enfoque que predominó durante gran parte de los años de la guerra fría.

POLITICA EXTERIOR DE SEGURIDAD ESTADOUNIDENSE EN LA POST GUERRA FRÍA Y EL MUNDO POST 11 DE SEPTIEMBRE 
Por otra parte, existe una visión más amplia con aspectos políticos, económicos y domésticos, Ilamada visión ampliacionista y que toma como variables en la formulación de la agenda de seguridad, a los intereses y valores de la cultura política estadounidense (supervivencia, justicia, libertad, democracia, prosperidad, prestigio y promoción de la ideología nacional), además de evaluar el medio internacional en los aspectos militar, político, económico y tecnológico, junto con la evaluación del medio doméstico en sus dimensiones económica, política y social.

Debido a que esta última visión sobre seguridad nacional permite un análisis multicausal, en el cual se identifican múltiples amenazas que provienen del área económica o política, a diferencia de la visión predominante en la guerra fría, cuando se identificaba a la Unión Soviética como la única fuente de amenazas, es la visión que encaja mejor en del escenario de posguerra fría, lleno de factores y elementos múltiples que desbordan los tópicos de análisis tradicionales y hace compleja la comprensión y la elaboración de la agenda exterior de seguridad estadounidense.

\subsection{La Estrategia Nacional de Seguridad Estadounidense en los años 90}

Para encontrar un punto de referencia, desde el cual se observe la agenda exterior de seguridad estadounidense, es imprescindible referirse a la Estrategia Nacional de Seguridad que por ser una directriz presidencial, es el marco de donde se desprende el resto de acciones sobre seguridad exterior y donde se delimitan las amenazas que el contexto internacional ofrece a Estados Unidos.

Como ya se mencionó, la agenda post guerra fría se amplió en términos militares, políticos, económicos y sociales, y trataba sobre múltiples y nuevos actores e incluso, sobre una nueva organización de los valores propios de la cultura política estadounidense. Ya no existía una sola amenaza que proveía otras amenazas secundarias, sino que por el contrario, durante los años 90, existieron amenazas múltiples que provenían de diferentes sectores (Powell, 93:35).

La Estrategia Nacional de Seguridad, desde 1997 hasta el 2001, propuso directrices muy similares, y su organización fue casi la misma. Sus objetivos centrales eran incrementar la seguridad de Estados Unidos mediante unas Fuerzas Armadas listas y equipadas para combatir, reforzar la prosperidad 
económica estadounidense y promover la democracia y la libertad, como los principales valores de la cultura política estadounidense.

De tal manera que la Estrategia Nacional de Seguridad se construyó sobre tres pilares: modelar el medio ambiente internacional; responder al espectro completo de las crisis; y prepararse para un futuro incierto.

El primer pilar de la Estrategia de Defensa, Modelar el medio ambiente internacional, buscaba con la diplomacia, el comercio y la inversión, promover y proteger los intereses nacionales de Estados Unidos, por medio del ordenamiento del escenario internacional. En otras palabras, el sistema internacional, después del fin de la guerra fría, era una masa amorfa, presta a tomar forma para servir a los intereses vitales y no vitales de Estados Unidos.

Para desarrollar este primer pilar, Estados Unidos disponía, en términos militares, de fuerzas estacionadas permanentemente en el exterior, fuerzas rotativamente desplegadas fuera de sus fronteras, fuerzas desplegadas temporalmente para ejercicios, entrenamiento combinado o intercambios entre militares, programas de cooperación en defensa, seguridad y programas de armamentos, actividades de asistencia humanitaria en conjunto con operaciones y ejercicios militares (Loh, 1994: 11-13; Khalilzad, 1997:52-58; Clarke, O'Connor, 1993: 442-457; Cohen, 2001). En América Latina, se conoció el Comando Sur, los ejercicios UNITAS, los programas de entrenamiento IMET, IMET ampliado y No IMET, una serie de políticas específicas basadas en el uso de Bases de Operaciones de Avanzada (FOL), ubicadas especialmente en la cuenca del Mar Caribe, así como contratistas.

En términos económicos, Estados Unidos desarrolló una agresiva política de alianzas y convenios económicos, especialmente con América. Las iniciativas de NAFTA y ALCA, tuvieron una importante visibilidad y protagonismo, y sólo se consumó la primera. De igual manera, se desarrolló un importante esquema de inversiones estadounidenses en varios países americanos y se profundizaron algunos diálogos políticos.

El segundo pilar de la Estrategia de Defensa, Responder al espectro completo de las crisis, puso de manifiesto la necesidad de usar los medios militares para responder a crisis que arriesgaran de alguna forma, los intereses de Estados Unidos en el mundo.

POLITICA EXTERIOR DE SEGURIDAD ESTADOUNIDENSE EN LA POST GUERRA FRÍA Y EL MUNDO POST 11 DE SEPTIEMBRE 
Este pilar descansaba en tres elementos: determinación de agresiones y coerciones en crisis; conducción de operaciones de contingencia en pequeña escala; y asegurar la victoria en los más importantes teatros de operaciones.

El primero de estos elementos, la determinación de agresiones y coerciones en crisis, implicó flexibilizar a las Fuerzas Militares de manera que su despliegue se realizara de manera ágil hacia el punto necesario, lo mismo que en áreas cercanas a la región donde se presentaba el problema. Este elemento contó aún con matices de guerra fría, al concebir la movilización de importantes fuerzas, en cuanto a su número, provenientes de los diferentes comandos unificados o de las flotas navales estacionadas en diferentes regiones del mundo, según los requerimientos estratégicos que se tenían en cuenta ante un enfrentamiento con las fuerzas del Pacto de Varsovia.

La conducción de operaciones de contingencia en pequeña escala (SSC), implicó preparar las fuerzas militares estadounidenses para guerras no convencionales o para conflictos de baja intensidad (Crane, 2001:1-4), constantes en América Central y en la terminación del conflicto colombiano (Bermúdez, 1989). Dentro de las SSC se incluyeron actividades como demostraciones de fuerza, campañas coercitivas, golpes limitados, operaciones de evacuación de no combatientes, vigilancia de zonas de restricción aérea, vigilancia de sanciones marítimas, operaciones de control de migraciones en masa, operaciones en contra del terrorismo, asistencia humanitaria, antinarcóticos y operaciones extranjeras en soporte de otras agencias gubernamentales de Estados Unidos. La participación de Estados Unidos en las SSC se propuso de manera selectiva, dependiendo de los intereses que tuviera el Estado o la región afectada, los costos y riesgos de la operación y la opción de usar otros medios diplomáticos. Se puede ubicar varios ejemplos de operaciones SSC en América Latina: la invasión de Grenada y la operación de captura del general Rafael Noriega en Panamá, fueron acciones típicas.

El último pilar de la Estrategia de Seguridad fue Prepararse para un futuro incierto. Tal y como su nombre lo sugiere, abría una gran puerta a una zona gris, sin alguna definición precisa sobre riesgos o amenazas. En este pilar, se preparó la respuesta a posibles amenazas múltiples que podían provenir de Rusia, Corea del Norte, China o Irak, en primer lugar, tales como proliferación de tecnología militar convencional y no convencional, y competencia económica, política y/o cultural. América Latina ofrecía otras amenazas 
REVISTA DE RELACIONES INTERNACIONALES, ESTRATEGIAY YEGURIDAD

múltiples e inciertas, aunque nunca de primer orden, como narcotráfico, migraciones, Estados débiles o inviables, asuntos medio ambientales y regímenes autoritarios.

Es importante destacar que el terrorismo no aparecía en esta agenda, como surgió con el ataque al World Trade Center y al Pentágono. Sólo se consideraba la posibilidad de ataques terroristas orientados y aplicados por enemigos estatales de Estados Unidos, tal vez porque esta agenda no logró adecuarse a un mundo sin Guerra Fría, y aún utilizaba categorías propias de este momento histórico, tales como disuasión y enemigo estatal, entre otras. En otras palabras, puesto que aún contenía y entendía un mundo bajo la guerra fría, no podía entender y enfrentar amenazas reales.

\section{LA NUEVA CONCEPCIÓN DE SEGURIDAD ESTADOUNIDENSE LUEGO DEL 11 DE SEPTIEMBRE DE 2001 Y SU IMPACTO EN AMÉRICA LATINA}

Ante la evidencia de la derrota de la Estrategia de Defensa estadounidense, luego de los ataques del 11 de septiembre de 2001, habría sido consecuente la construcción de una agenda radicalmente nueva, en la cual se identificaran las amenazas reales que enfrentaba Estados Unidos.

Se hicieron cambios, especialmente en el entendimiento de la seguridad y la percepción de amenazas. Sin embargo, se puede afirmar que, contrariamente a lo que se ve a simple vista, la actual agenda exterior de seguridad de Estados Unidos, poco difiere de la agenda que fue derrotada con el ataque al World Trade Center y al Pentágono.

\subsection{La seguridad preventiva. Nuevo concepto para una vieja amenaza}

Así como el cambio que se produjo en el sistema internacional luego del fin de la Guerra Fría produjo un debate en el entendimiento y conceptualización de la seguridad, en lo que va corrido del siglo XXI un nuevo concepto ha sido construido y aplicado para dirigir las acciones de defensa de Estados Unidos.

Se puede afirmar entonces que desde una visión tradicional de la seguridad, en la guerra fría, se pasó a una visión ampliacionista en los años 90, y

POLITICA EXTERIOR DE SEGURIDAD ESTADOUNIDENSE EN LA POST GUERRA FRÍA Y EL MUNDO POST 11 DE SEPTIEMBRE 
luego del ataque del 11 de septiembre de 2001, se desarrolló la visión preventiva de seguridad.

Esta concepción de la seguridad plantea en grandes rasgos, la necesidad de emprender acciones primordialmente militares o políticas en contra de fuentes de amenazas, sean Estados hostiles o Estados hospederos de agrupaciones terroristas, aunque la amenaza no se haya establecido por completo. Esto implica que la mejor forma de sostener la integridad del Estado es actuar militarmente en contra de las amenazas, incluso antes de que éstas se consoliden.

En este orden de ideas, resulta especialmente caprichoso identificar una amenaza o una fuente de amenazas, pues la percepción del riesgo puede no estar del todo identificada y se puede sacrificar la legalidad internacional y la legitimidad interna. De hecho, en Naciones Unidas se cuestionó el seguimiento de la idea de seguridad preventiva, debido a que sólo se considera legal un ataque, siempre y cuando se responda a una acción hostil, militar, política, económica o social consumada por parte de un Estado o agrupación hostil. La segunda guerra de Irak, por ejemplo, no gozó del respaldo del sistema ONU y se le calificó de ilegal dentro del derecho internacional, por soportarse en sospechas, infundadas por demás, de una amenaza que no había dado muestras de existir realmente y sin haberse dado alguna acción militar o política provocativa por parte del régimen de Saddam. De igual forma, la etérea identificación de las amenazas que se maneja en el concepto de Seguridad Preventiva, produjo salidas en falso para legitimar las acciones armadas de Estados Unidos, dentro de su sociedad y su opinión pública. El argumento que inicialmente se expuso, la búsqueda y destrucción del arsenal de destrucción masiva de Irak, tuvo que ser cambiado sobre la marcha de los acontecimientos y modificado por la búsqueda de la democracia en Irak o la búsqueda de las libertades civiles en Irak o simplemente, la búsqueda de la estabilidad regional. No obstante, y como producto de la improvisación política y militar que puede caracterizar a la Seguridad Preventiva, ninguna de las justificaciones con las que se quiso legitimar la guerra contra Irak se ha consumado, y en cambio acarreó serios costos políticos a la administración Bush.

Surge entonces, la inquietud de saber cuál es el criterio para definir una amenaza en gestación. En términos académicos, es simple y preocupante porque la respuesta carece de toda rigurosidad metodológica; básicamente, 
y como se expresa en la Estrategia Nacional de Seguridad desde octubre de 2001, toda acción que atente contra los intereses vitales o no vitales de Estados Unidos ${ }^{2}$, se considera una amenaza a la seguridad nacional y toda organización que se encargue de ejecutarla, es en consecuencia, una agrupación terrorista.

\subsection{La Estrategia Nacional de Seguridad Estadounidense Contemporánea}

En principio, después de los ataques al World Trade Center, se diseñó una agenda exterior de seguridad estadounidense nueva y acorde con las amenazas evidentes. Así se establecieron una serie nueva de pilares de la seguridad que ampliaban aún más el espectro de las amenazas. En efecto, los elementos más importantes de esta nueva estrategia son en primer lugar, la lucha antiterrorista mundial, soportada en una serie de alianzas (QDRR, 2001), que recordaban en alguna medida, la distinción amigo - enemigo propia de la guerra fría.

En esta renovada división del mundo, América Latina parece alinearse, al menos parcialmente, con Estados Unidos. Centroamérica, pendiente de una importante negociación económica que propone un área de libre comercio con Estados Unidos, ofrece un respaldo constante a la nominación de terroristas que toman otros regímenes, a la luz de los intereses estadounidenses. Esta estrecha relación económica, se ve profundizada por la estructura de Bases de Operaciones de Avanzada (FOL), desplegada en Honduras, Nicaragua y El Salvador desde los años de la guerra fría, y que se convierten en importantes centros de entrenamiento militar de unidades de guerra especial, unidades específicas en guerra irregular empleadas en el desarrollo de operaciones de contingencia de pequeña escala (Bermúdez, 1989).

Colombia es tal vez, el país americano más comprometido con esta nueva doctrina de defensa estadounidense. Esta cercanía resulta muy conveniente a partir de criterios políticos inherentes al conflicto armado interno, pues el

\footnotetext{
2 Se entiende como Intereses Nacional Vitales, los elementos en los cuales se sostiene el Estado y cuyo ataque comprometería la supervivencia del mismo; por ejemplo: la vida y seguridad de los ciudadanos estadounidenses dentro y fuera del territorio nacional. En cuanto a los intereses No Vitales pero Importantes, se pueden definir como los que no comprometen la supervivencia del Estado en sí, sino su status de potencia mundial; por ejemplo: el acceso a recursos energéticos y petroleros, y a mercados importantes.
}

POLITICA EXTERIOR DE SEGURIDAD ESTADOUNIDENSE EN LA POST GUERRA FRÍA Y EL MUNDO POST 11 DE SEPTIEMBRE 
gobierno colombiano se alimenta de la confusa conceptualización que ofrece el término terrorista y la aplica, al igual que Estados Unidos, según sus conveniencias. Esto se puede observar en la evolución del discurso antisubversivo generado desde el gobierno colombiano, que pasó de nominar a los grupos irregulares subversivos como organizaciones bandoleras y comunistas, en los años de la guerra fría, a organizaciones narcotraficantes en los años 90 y por último, organizaciones terroristas en la actualidad.

La utilidad de esta nominación no es solamente discursiva, sino que refleja una sincronía con los pilares de defensa estadounidenses, de manera que la lucha colombiana contra la subversión, logra un apoyo inmediato desde Estados Unidos en los campos militar, político y económico. En el aspecto internacional, el acompañamiento colombiano a las iniciativas estadounidenses, tiene un impacto residual, y sin embargo, el presidente Álvaro Uribe ha manifestado públicamente su apoyo, desde la primera reacción militar a los ataques de 2001, como fue la campaña de Afganistán.

No obstante, América Latina también plantea elementos en discordia con esta lógica antiterrorista estadounidense. El caso más claro es Venezuela, donde el régimen de Hugo Chávez, no sólo nunca ha avalado las intervenciones militares de Estados Unidos y las califica incluso de ocupaciones o invasiones, sino que ha adelantado una dinámica diplomacia con regímenes hostiles o abiertamente alejados de Estados Unidos. Esta diplomacia además de perfilar un bloque de oposición política, ha representado para Venezuela varios elementos importantes. En primer lugar, ha logrado una serie de acuerdos de compras militares que lo impulsan de nuevo a su antigua condición de primacía militar (en términos tecnológicos y materiales), suramericana y andina, posición que compartía históricamente con Perú y Brasil. En segundo lugar, le ha permitido afianzar su liderazgo político y estratégico - militar en la región andina, con acuerdos como el firmado recientemente con Bolivia, en el cual se compromete, entre otras cosas, a asesorar al gobierno boliviano en la readecuación de su estructura de defensa, mediante un número importante de bases militares y puestos de frontera. En tercer lugar, le ha permitido a Venezuela proponerse como uno de los abanderados en una nueva instancia política internacional que cumpla un papel similar al que quiso cumplir los NOAL en la guerra fría.

El segundo elemento de la agenda exterior de seguridad estadounidense en la coyuntura antiterrorista, es impedir amenazas con armas de destrucción 
masiva. Este pilar es concordante con el referido a la acción y delimitación antiterrorista, puesto que se convierte en el argumento material de la acción militar. Sin embargo, resulta paradójico que siga la misma lógica estratégica que proponía la agenda anterior. En ella, se identificaba igualmente como amenazas, la proliferación de tecnología militar en particular, armamento nuclear, mientras que en la actual se renombra simplemente como armas de destrucción masiva.

El tercer pilar de la nueva estrategia, se refiere a la necesidad de impedir o resolver prontamente los conflictos regionales. Este pilar se asume como prioridad en la seguridad estadounidense, porque argumenta que un conflicto regional puede inducir a inestabilidades o dinamizar el comercio clandestino de material de guerra, ambos escenarios adecuados para el desarroIlo o proyección de grupos terroristas. En este punto, es importante mencionar que en la agenda actual de seguridad estadounidense, las agrupaciones terroristas sólo existen bajo una dimensión estatal, bien sea bajo un Estado hospedero o porque se apropian de un Estado débil. En un sentido académi$\mathrm{CO}$, resulta cuestionable esta idea porque niega la posibilidad de la existencia de agrupaciones terroristas deslocalizadas.

El cuarto y el quinto pilar en la agenda de seguridad actual, abarcan una dimensión humanista, los cuales son Abogar por la dignidad humana y Expandir la democracia. En términos de defensa, la razón de ser de estos dos pilares aparece cuando se habla de estabilidad regional que requiere alguna noción de derechos humanos, en este caso, la noción estadounidense, además de un sistema democrático que asegure transiciones en el ejercicio del poder y disminuya la posibilidad de que un enemigo se instituya en forma vitalicia, en el poder de algún país.

Por último, se plantea la necesidad de reformar las instituciones de seguridad, de manera que la acción de inteligencia y la acción de respuesta militar sean fluidas y se mantenga la supremacía militar de Estados Unidos en el mundo.

\section{A MANERA DE CONCLUSIÓN}

Una vez hecho el recorrido por la estructura de defensa estadounidense, luego de 2001, queda la impresión de que la agenda fue modificada 
sustancialmente. Sin embargo, si se retoman los pilares recién mencionados, se puede establecer un paralelo con la agenda de seguridad que estaba vigente luego del fin de la guerra fría y que fue derrotada con los ataques terroristas.

Por ejemplo, la búsqueda de estabilidad regional, presente en la actual agenda, también estaba presente en la anterior, cuando se hablaba de la necesidad de Modelar el medio ambiente internacional. De igual manera, el temor por la proliferación de armas de destrucción masiva, se reflejaba en la anterior agenda, cuando se identificaba como amenaza, la proliferación de tecnología nuclear y militar convencional. Se puede decir entonces, que la agenda exterior de seguridad de Estados Unidos sólo tuvo como modificación estructural, el hecho de identificar como amenaza primordial al terrorismo y establecer la necesidad de realizar varias acciones para combatirlo, modificación apenas obvia, si se tiene en cuenta que precisamente fue el terrorismo lo que motivó el supuesto cambio de agenda.

En este punto, América Latina otra vez queda relegada a un papel secundario en la lógica internacional - estratégica de Estados Unidos, rescatada solamente por intereses secundarios, prácticamente invisibles o por la conveniencia a la hora de utilizar el término de terrorismo como es el caso colombiano.

\section{BIBLIOGRAFÍA}

BAEUFRE, A. (1985). Introduction à la stratégie. Institute Français des Relations Internationales. Paris: Economique.

BÁRCENAS, M. (2000). La reconceptualización de la seguridad: el debate contemporáneo. En: Revista Mexicana de Política Exterior, 59. México.

BERMÚDEZ, L. (1989). Guerra de baja intensidad. Reagan contra Centroamérica. México: Siglo XXI.

BUZAN, B. (1993). From International System to International Society: Structural Realism and regime theory meet the English School. En: International Organization, 47.

CLARKE, D. y O 'CONNOR, D. (1993). US Base-Rigts payments after the cold war. En: Orbis. Vol. 37, 3. Summer. 
COHEN, E. (2001). El sistema de defensa estadounidense en el siglo XXI. En: Foreign Affairs en Español. Primavera.

COLLIN, P. (Dic. 92/93). US Forces: Challenges Ahead. En: Foreign Affairs, 5.

CRANE, C. (2001). Landpower and crises: Army roles and misions in smaller - scale contingencies during the 1990. US Army War College. Strategic Studies Institute.

DEPARTMENT OF DEFENSE. UNITED STATES OF AMERICA. Quadrennial Defense Review Report. 30 september, 2001.

FONTANA, Andrés. (1997). Complejidad de riesgo e interdependencia, tendencias de cambio en la seguridad internacional. Serie Documentos de trabajo, 24. Buenos Aires: Instituto del Servicio Exterior de la Nación.

INICIATIVA DE DEFENSA ESTRATÉGICA. Informe sobre Seguridad Nacional del Presidente de los Estados Unidos de Norteamérica al Congreso de la Unión. Washington: 2001.

KAUFFMAN, D., McKITRICK, J., y LENEY, T. (1985). How to Analyze National Security, en Kauffman D. McKitrick J, y Leney T (Comps). US National Security. A Framework for Analysis. Lexington, Lexington Books.

KHALILZAD, Z. y OCHMANEK, D. (1997). Rethinking US defense planning. En: Survival. Vol. 39.

LOH, John. (1994). Adapting US military organizations to the new security environment. En: Strategic Review. Vol. 22, 2.

SWEEDLER, A. (1999). La política de Seguridad de Estados Unidos en la post guerra fría. En: Revista Estudios Internacionales. Año XXV, 99.

WALTZ, K. (1988). Teoría de la Política Internacional. Colección Estudios Internacionales. Buenos Aires: Grupo Editor Latinoamericano.

POLÍTICA EXTERIOR DE SEGURIDAD ESTADOUNIDENSE EN LA POST GUERRA FRÍA Y EL MUNDO POST 11 DE SEPTIEMBRE 